Supporting information

\title{
Flexible Microswimmer Manipulation in Multiple Microfluidic Systems utilizing Thermal Buoyancy-Capillary Convection
}

\author{
Kailiang Zhang ${ }^{1}$, Yukun Ren ${ }^{* 1,2}$, Meiying Zhao ${ }^{1}$, Tianyi Jiang ${ }^{1}$, Likai Hou ${ }^{1}$, and Hongyuan Jiang ${ }^{* 1}$ \\ ${ }^{1}$ School of Mechatronics Engineering, Harbin Institute of Technology, Harbin, Heilongiiang, PR China 150001, Email: \\ rykhit@hit.edu.cn,jhy_hit@hit.edu.cn. \\ ${ }^{2}$ State Key Laboratory of Robotics and System, Harbin Institute of Technology, Harbin, Heilongiiang, PR China 150001.
}

\section{Electronic Supporting Information (ESI)}

Section S1. Key parameters of the microchip used for swimmer manipulation.

Section S2. Materials and microswimmer preparation.

Section S3. Theoretical background.

Section S4. Numerical simulation model and material properties.

\author{
Additional Supplementary Material (AVI): \\ Video S1: Swimming of PS microswimmers under the energization of one microheater \\ Video S2: Swimming of PS microswimmers under the energization of two adjacent microheaters \\ Video S3: Two-dimensional manipulation of PS microswimmers at a solid-liquid interface \\ Video S4: Swimming of BA microswimmers at a gas-liquid interface \\ Video S5: Swimming of TMPTA microswimmers under the modulation of one microheater \\ Video S6: Swimming of TMPTA microswimmers under the energization of multiple microheaters
}




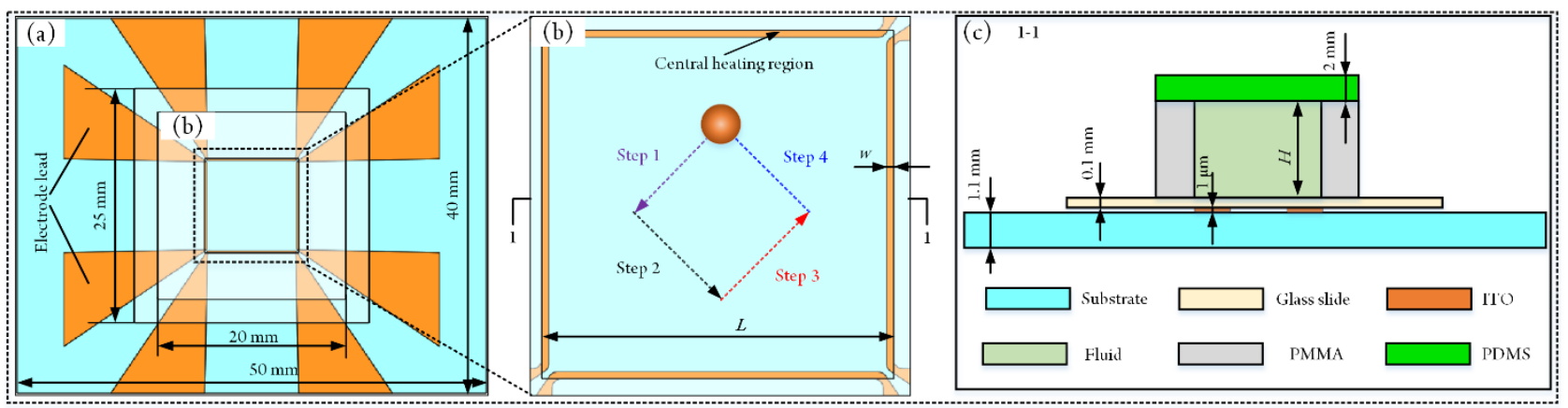

Figure S1. (a-b) Top view of the Microdevice. (c) Side view of the microchip.

Table S1. Key parameters of the microchip

\begin{tabular}{|c|c|c|c|}
\hline Parameters & Channel size $L$ & Microheater width $w$ & Channel height $H$ \\
\hline Device for PS microswimmer control & $3 \mathrm{~mm}$ & $0.06 \mathrm{~mm}$ & $1.4 \mathrm{~mm}$ \\
\hline Device for BA and TMPTA microswimmers & $10 \mathrm{~mm}$ & $0.2 \mathrm{~mm}$ & $1,3,6 \mathrm{~mm}$ \\
\hline
\end{tabular}

\section{Section S2: Materials and Microswimmer Preparation}

Materials and Devices. The 20- $\mu \mathrm{m}$ polysterene microspheres were purchased from Tianjin Baseline ChromTec Research Center (Tianjin, China). Poly (vinyl alcohol) (PVA), ethoxylated (3) trimethylolpropane triacrylate (EO3 TMPTA), 2hydroxy-2-methylpropiophenone (HMPP) were obtained from Sigma-Aldrich. Butyl acrylate (BA), methylene blue and Nile red were bought from Aladdin (Shanghai, China).

Two syringe pumps (PHD Ultra, Harvard Apparatus) are used to inject solutions into glass capillaries. Four direct current power supplies (KXN-305D, Zhao Xin, China) are utilized to energize the microheaters. The experiments are visualized through an inversed microscope (IX73, Olympus, Japan) with a CMOS camera (Prime 95B, Photometrics).

Preparation of BA and TMPTA Microswimmers. To generate BA microswimmers, a capillary microdevice is constructed by using glass capillary tubes (World precision instruments, Inc.). Specifically, a square capillary (inner size 1.03mm) is settled on a glass slide. Two tapered cylindrical tubes (inner diameter (ID) $0.58 \mathrm{~mm}$, outer diameter (OD) $1.03 \mathrm{~mm}$ ) are coaxially assembled in the opposite ends of the square capillary (AIT Glass, Inc., 810-9917) with an axial spacing of $60 \mu \mathrm{m}$. And the inner diameters of tapered orifices of these two cylindrical capillaries are $40 \mu \mathrm{m}$ and $90 \mu \mathrm{m}$, respectively. When preparing BA microspheres by using the device, the outer phase is an aqueous solution with $5 \mathrm{wt} \%$ PVA. The inner phase is $99 \mathrm{wt} \%$ BA mixed with $1 \mathrm{wt} \%$ HMPP, and the solution is dyed by using Nile red. The volume flow rates of the inner and outer phases are $500 \mathrm{uL} / \mathrm{h}$ and $2 \mathrm{~mL} / \mathrm{h}$ respectively. The generated droplet swimmers are solidified by the lighting of an ultraviolet lamp (HTLD-4II, Shenzhen zhijun photoelectric co., LTD, China). The generation process of TMPTA microswimmers is similar to that of BA droplets. The only difference is that the outer $5 \mathrm{wt} \%$ PVA solution has a flow rate of $10 \mathrm{~mL} / \mathrm{h}$, and the inner phase is a mixture of $99 \mathrm{wt} \%$ TMPTA and $1 \mathrm{wt} \%$ HMPP with a flow rate of $500 \mathrm{uL} / \mathrm{h}$.

\section{Section S3: Theoretical background}

The basic theories in our study include three parts: heat transfer in microchip, fluid convection and swimmer swimming.

1. Heat transfer. Four ITO-made microheaters are the heating sources of the microsystem, and their temperature distributions meet the following equation,

$$
\rho_{I} c_{I} \frac{\partial T}{\partial t}=\nabla \cdot\left(k_{I} \nabla T\right)+q
$$

where, $\rho_{I}$ is the density of ITO film, $c_{I}$ the heat capacity, $k_{I}$ the thermal conductivity, $q$ the Joule heating source. 
The Joule heating flux density $q$ is determined by the electric current density and electric field intensity,

$$
q=\boldsymbol{J} \cdot \boldsymbol{E}=\sigma \cdot|\nabla \phi|^{2}
$$

where, $q$ is the Joule heat flux density, $J$ the electric current density, $E$ the electric field intensity, $\sigma$ the conductivity of ITO film. $\phi$ the electric potential. $\phi$ satisfies $\nabla \cdot(\sigma \boldsymbol{E})=-\sigma \nabla^{2} \phi=0$.

At the microheater's surface, the normal electric current density $J_{n}$ is 0 .

As for the other solid materials of the microchip, their temperature distributions satisfy the unsteady-state heatconduction differential equation without internal heat source,

$$
\rho_{g p} c_{g p} \frac{\partial T}{\partial t}=\nabla \cdot\left(k_{g p} \nabla T\right)
$$

where, $\rho_{g p}$ is the density, $c_{g p}$ the heat capacity, $k_{g p}$ the thermal conductivity.

The heat transfer in fluids meets,

$$
\rho_{0} c_{p}\left(\frac{\partial T}{\partial t}+u \cdot \nabla T\right)-\nabla \cdot\left(k_{f} \nabla T\right)=0
$$

where, $\rho_{0}$ is the density, $c_{p}$ the heat capacity, $k_{f}$ the thermal conductivity.

At the interfaces of different materials, the temperature and the normal heat flux are continuous.

The chip surface-air satisfies,

$$
-k\left(\frac{\partial T}{\partial n}\right)_{s}=h\left(T_{s}-T_{r}\right)
$$

where, $k$ is the thermal conductivity, $h$ the convective heat transfer coefficient, $n$ the external normal line of the outer surface of the microchip, $T_{s}$ the temperature of the chip's surface, $T_{r}$ the temperature of the surrounding air.

2. Fluid convection. The buoyancy-capillary convection is governed by the Navier-Stokes and the continuity equations.

$$
\begin{gathered}
\rho_{0}(\boldsymbol{u} \cdot \nabla) \boldsymbol{u}=\mu(T) \nabla^{2} \boldsymbol{u}-\nabla p+\boldsymbol{F}_{b}+\boldsymbol{F}_{c} \\
\nabla \cdot \boldsymbol{u}=0
\end{gathered}
$$

where, $\rho_{0}$ is the fluid density at room temperature $T_{0}, \mu(T)$ the dynamic viscosity at temperature $T, p$ the pressure, $\boldsymbol{F}_{b}$ the buoyancy force, $\boldsymbol{F}_{b}=-\boldsymbol{g}\left[\rho_{0}-\rho(T)\right] \approx-\boldsymbol{g} \rho_{0} \beta_{T}\left(T-T_{0}\right)\left(\rho(T)\right.$ the fluid density at temperature $T$, $\beta_{T}$ the thermal expansion coefficient). $\quad \boldsymbol{F}_{c}$ the thermo-capillary stress, $\boldsymbol{F}_{c}=-\sigma_{T} \nabla_{S} T\left(\sigma_{T}\right.$ the temperature coefficient of surface tension, $\nabla_{S} T$ the tangential temperature gradient at fluidic interface).

At fluid-wall interfaces, the flow field meets the no-slip boundary condition: $\boldsymbol{u}_{\text {wall }}=0$.

3. Swimmer swimming. The forces acting on microswimmers include Stokes drag, gravity force, buoyancy force (sustaining strength), and thermophoretic force. The movement of microswimmers at the bottom of a fluid, the gasliquid interface and the liquid interface will be affected by fluid convection and tangential thermophoretic force.

The microswimmer velocity induced by thermophoretic force $\boldsymbol{F}_{T}$ is linear to the temperature gradient:

$$
v_{T}=-D_{T} \cdot \nabla T
$$

where, $D_{T}$ is the thermal diffusion coefficient. The magnitude of $D_{T}$ in wide range of soft colloidal systems (e.g. polymers, proteins, DNA) is relatively unchanging, staying within the range $10^{-8} \mathrm{~cm}^{2} / \mathrm{Ks} \sim 10^{-7} \mathrm{~cm}^{2} / \mathrm{Ks}^{1,2}$. The $D_{T}$ with a value of $1 \times 10^{-7} \mathrm{~cm}^{2} / \mathrm{Ks}$ is selected to evaluate the thermophoretic velocity of particle in our analysis.

Therefore, the migration velocity of microswimmer caused by fluid convection and thermophoresis is,

$$
\boldsymbol{v}_{p}=\boldsymbol{u}+\boldsymbol{v}_{T}
$$

\section{Section S4: Numerical simulation model and material properties.}

To conduct numerical analyses, a 3-dimensional geometrical model is first established in a software of Solidworks 2014 (Figure S2). Then the model is imported in a Comsol software (COMSOL Multiphysics 5.5). The physical model of the microsystem includes three parts: electric current, heat transfer, and fluid convection, and these basic theories are given in Section S3 of the Supporting Information. According to this, we set different boundaries conditions and governing equations 
for different parts of the numerical model, as shown in Table S2. By solving above equations using the Comsol software, we finally obtain the temperature and convection flow of the microdevice. The material properties used in the simulation are provided Table S3.

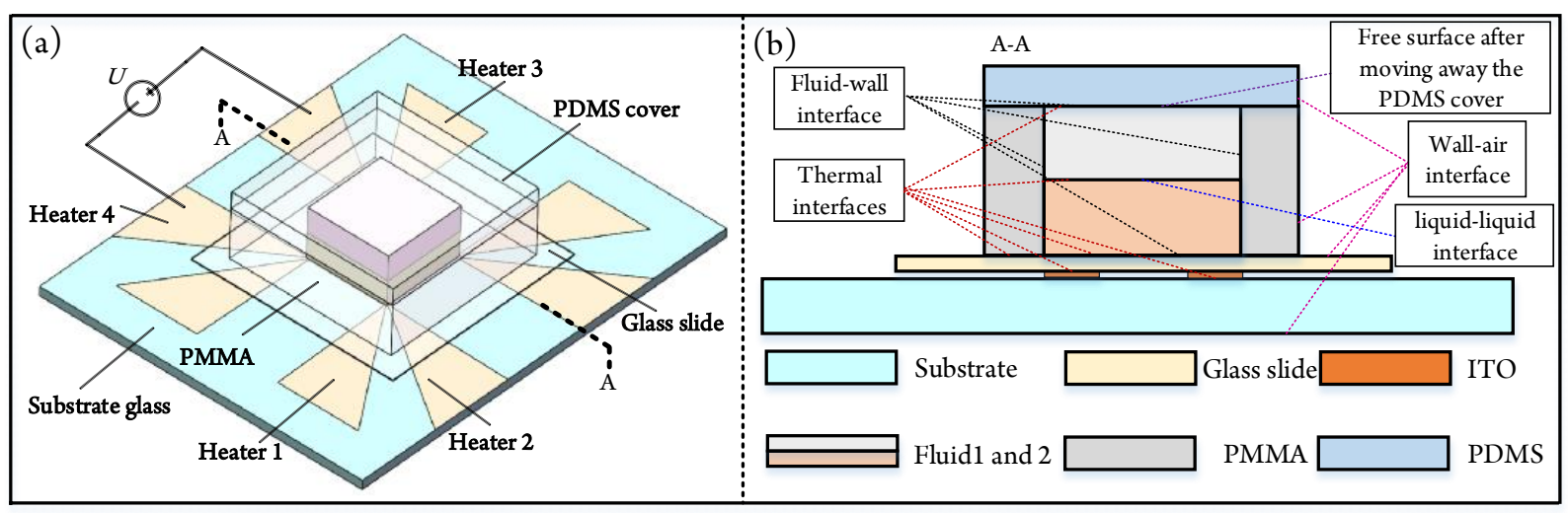

Figure S2. (a) Three-dimensional geometrical model. (b) Side view of the microdevice.

Table S2. Governing equations and boundary conditions used in the numerical simulation.

\begin{tabular}{|c|c|c|c|}
\hline Position & Electric field & Thermal field & Flow field \\
\hline Microheaters & $\nabla \cdot(\sigma E)=-\sigma \nabla^{2} \phi=0$ & $\rho_{e} \mathcal{c}_{e} \frac{\partial T}{\partial t}=\nabla \cdot\left(k_{e} \nabla T\right)+\sigma \cdot|\nabla \phi|^{2}$ & - - \\
\hline $\begin{array}{l}\text { Surfaces of } \\
\text { microheaters }\end{array}$ & $\boldsymbol{n} \cdot \nabla \phi=0$ & $\left\{\begin{array}{c}T_{i}=T_{j} \\
\Phi_{i}=\Phi_{j}\end{array}\right.$ & - - \\
\hline $\begin{array}{l}\text { Glass substrate } \\
\text { and glass slide }\end{array}$ & - - & $\rho_{g} c_{g} \frac{\partial T}{\partial t}=\nabla \cdot\left(k_{g} \nabla T\right)$ & - - \\
\hline PMMA channel & - - & $\rho_{p \mathrm{~m}} c_{p m} \frac{\partial T}{\partial t}=\nabla \cdot\left(k_{p m} \nabla T\right)$ & - - \\
\hline PDMS cover & - & $\rho_{p d} \mathcal{c}_{p d} \frac{\partial T}{\partial t}=\nabla \cdot\left(k_{p d} \nabla T\right)$ & - - \\
\hline Fluids & - & $\rho_{0} c_{p}\left(\frac{\partial T}{\partial t}+u \cdot \nabla T\right)-\nabla \cdot\left(k_{f} \nabla T\right)=0$ & $\left\{\begin{array}{c}\rho_{0}(\boldsymbol{u} \cdot \nabla) \boldsymbol{u}=\mu(T) \nabla^{2} \boldsymbol{u}-\nabla p+\boldsymbol{F}_{b} \\
\nabla \cdot \boldsymbol{u}=0\end{array}\right.$ \\
\hline $\begin{array}{l}\text { Solid-solid } \\
\text { interfaces }\end{array}$ & & $\left\{\begin{array}{c}T_{i}=T_{j} \\
\Phi_{i}=\Phi_{j}\end{array}\right.$ & - \\
\hline $\begin{array}{l}\text { Solid-liquid } \\
\text { interfaces }\end{array}$ & & $\left\{\begin{array}{c}T_{i}=T_{j} \\
\Phi_{i}=\Phi_{j}\end{array}\right.$ & $\boldsymbol{u}=0$ \\
\hline $\begin{array}{l}\text { Gas-liquid } \\
\text { interfaces }\end{array}$ & 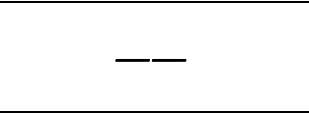 & $-k\left(\frac{\partial T}{\partial n}\right)_{w}=h\left(T_{w}-T_{a}\right)$ & $\begin{array}{l}{\left[\mu\left(\nabla \boldsymbol{u}+(\nabla \boldsymbol{u})^{\mathbf{T}}\right)\right] \boldsymbol{n}=\nabla_{\mathbf{t}} \gamma} \\
=\nabla \gamma-\boldsymbol{n} \cdot(\boldsymbol{n} \cdot \nabla \gamma)\end{array}$ \\
\hline $\begin{array}{l}\text { Liquid-liquid } \\
\text { interfaces }\end{array}$ & & $\left\{\begin{array}{c}T_{i}=T_{j} \\
\Phi_{i}=\Phi_{j}\end{array}\right.$ & $\left\{\begin{array}{c}\boldsymbol{n}_{1}\left(\boldsymbol{T}_{1}-\boldsymbol{T}_{2}\right)=\gamma\left(\nabla_{\mathbf{t}} \cdot \boldsymbol{n}_{1}\right) \boldsymbol{n}_{1}-\nabla_{\mathbf{t}} \gamma \\
\boldsymbol{T}=-\mathbf{p} \boldsymbol{I}+\boldsymbol{K} \\
\boldsymbol{K}=\mu\left(\nabla \boldsymbol{u}+(\nabla \boldsymbol{u})^{\mathbf{T}}\right) \\
\boldsymbol{u}_{1}=\boldsymbol{u}_{2}\end{array}\right.$ \\
\hline Wall-air & - & $-k\left(\frac{\partial T}{\partial n}\right)_{w}=h\left(T_{w}-T_{a}\right)$ & - - \\
\hline
\end{tabular}


Notes: $\sigma$ is the conductivity of the microheater; $\phi$ is the electric potential; $\rho$ is the density; $c$ is the heat capacity; $k$ is the thermal conductivity; $T_{i}$ and $T_{j}$ are the temperatures of the two sides of the interfaces; $\Phi_{i}$ and $\Phi_{j}$ are the normal heat flux of the two sides of the interface; $\rho_{0}$ is the density of liquid media at room temperature; $u$ is the velocity; $\mu(T)$ is the dynamic fluid viscosity at temperature $T ; \quad p$ is the pressure; $F_{b}=-g\left(\rho_{0}-\rho\right) \approx-g \rho_{0} \beta_{T}\left(T-T_{0}\right)$ is the buoyancy force.

Table S3. Material properties

\begin{tabular}{|c|c|c|c|c|c|c|}
\hline & $\begin{array}{c}\text { Electric } \\
\text { conductivity } \\
{[\mathrm{S} / \mathrm{m}]}\end{array}$ & $\begin{array}{l}\text { Density } \\
{\left[\mathrm{kg} / \mathrm{m}^{3}\right]}\end{array}$ & $\begin{array}{c}\text { Heat } \\
\text { capacity } \\
{[\mathrm{J} /(\mathrm{kg} \cdot \mathrm{K})]}\end{array}$ & $\begin{array}{l}\text { Thermal } \\
\text { conductivity } \\
{[\mathrm{W} /(\mathrm{m} \cdot \mathrm{K})]}\end{array}$ & Viscosity & $\begin{array}{l}\text { Surface tension } \\
\qquad[\mathrm{mN} / \mathrm{m}]\end{array}$ \\
\hline Glass $^{3}$ & - - & 2203 & 703 & 1.38 & - - & - \\
\hline $\mathrm{PMMA}^{3}$ & - - & 1190 & 1420 & 0.19 & - & - \\
\hline $\begin{array}{c}\text { Solid } \\
\text { PDMS }^{3}\end{array}$ & & 970 & 1460 & 0.16 & - & - - \\
\hline Water ${ }^{3}$ & -- & $\begin{array}{c}744.145+1 \\
9447 \times T- \\
0.00368 \times T \\
2\end{array}$ & $\begin{array}{c}4035.841+0 \\
492312 \times T\end{array}$ & $\begin{array}{c}- \\
0.9004+0.00 \\
84 \times T- \\
1.1182 \times 10^{-} \\
{ }^{5} \times T^{2}\end{array}$ & $\begin{array}{c}0.381- \\
0.0044 \times T+1.9 \times \\
10^{-05} \times T^{2}- \\
3.6736 \times 10^{-} \\
{ }^{08} \mathrm{~T}^{3}+2.6667 \times 10^{-} \\
{ }^{11} \times T^{4}[\mathrm{~Pa} \cdot \mathrm{s}]\end{array}$ & $\begin{array}{c}77.18- \\
0.203 \times(T- \\
273.15)\end{array}$ \\
\hline $\mathrm{ITO}^{4}$ & $2.1 \times 10^{5}$ & 7179 & 1000 & 2 & - & - \\
\hline $\begin{array}{c}\text { HFE } \\
7500^{5,6}\end{array}$ & & $\begin{array}{c}1665.8- \\
2.0845 \times(T \\
-273.15)\end{array}$ & $\begin{array}{c}1.4982 \times(T- \\
273.15)+109 \\
1\end{array}$ & $\begin{array}{c}0.069- \\
1.798 \times 10^{-} \\
{ }^{4} \times(T- \\
273.15)+4.2 \\
4 \times 10^{-7} \times(\mathrm{T}- \\
273.15)^{2}\end{array}$ & $\begin{array}{c}75.23 \times \exp (- \\
1883.93 \times T^{1}- \\
0.00819 \times T+368 \\
\left.523 \times T^{2}\right) \\
{\left[\mathrm{mm}^{2} / \mathrm{s}\right]}\end{array}$ & $\begin{array}{c}52.165 \times(1- \\
T / 534.15)^{1.26} \times \\
{[1-0.2832 \times(1-} \\
\left.T / 534.15)^{0.5}\right]\end{array}$ \\
\hline $\begin{array}{c}\text { Silicone } \\
\text { oil }^{7-9}\end{array}$ & & $\begin{array}{c}1118.9881 \\
6- \\
1.02082 \times T\end{array}$ & 2090 & 0.14 & $\begin{array}{c}0.65 \times(4.3004- \\
0.01148 \times T) \\
{[\mathrm{mPa} \cdot \mathrm{s}]}\end{array}$ & $\begin{array}{l}37.12616- \\
0.06222 \times T\end{array}$ \\
\hline
\end{tabular}

Notes: $T[\mathrm{~K}]$ is the temperature.

\section{References:}

(1) Würger A. Transport in charged colloids driven by thermoelectricity. Physical review letters, 2008, 101(10): 108302.

(2) Talbot E L, Kotar J, Parolini L, et al. Thermophoretic migration of vesicles depends on mean temperature and head group chemistry. Nature communications, 2017, 8(1): 1-8.

(3) From the Material Library of Comsol software.

(4) Provided by the South China Science \& Technology Company, China. 
(5) Rausch M H, Kretschmer L, Will S, et al. Density, surface tension, and kinematic viscosity of hydrofluoroethers HFE7000, HFE-7100, HFE-7200, HFE-7300, and HFE-7500. Journal of chemical \& engineering data, 2015, 60(12): 3759-3765.

(6) Bi S , Cui J , Ma L , et al. Thermophysical properties of HFE7100 and HFE7500. Ciesc Journal, 2016.

(7) Ancherbak S, Yasnou V, Mialdun A, et al. Coexistence curve, density, and viscosity for the binary system of perfluorohexane+ silicone oil. Journal of Chemical \& Engineering Data, 2018, 63(8): 3008-3017.

(8) Ricci E, Sangiorgi R, Passerone A. Density and surface tension of dioctylphthalate, silicone oil and their solutions. Surface and Coatings Technology, 1986, 28(2): 215-223.

(9) http://cn.comsol.com/model/marangoni-effect-20329. 